Original Research

\title{
Injuries in Quidditch: A Prospective Study from a Complete UK
}

\section{Season}

Rachel Pennington ${ }^{1}$ (1) ${ }^{2}$, Ashley Cooper ${ }^{2}$, Alastair C Faulkner ${ }^{3}$, Alasdair MacInnes ${ }^{3}$, Thomas S W Greensmith ${ }^{4} \mathbb{0}$, Alistair $^{2}$ I W Mayne ${ }^{5}$, Peter S E Davies ${ }^{3}$ (1)

${ }^{1}$ St Andrews University, Scotland, ${ }^{2}$ Psychiatry, Pennine Care NHS Foundation Trust, UK, ${ }^{3}$ Trauma and Orthopaedics, NHS Tayside, Dundee, Scotland, ${ }^{4}$ Trauma and Orthopaedics, County Durham and Darlington NHS Foundation Trust, UK, 5 Trauma and Orthopaedics, Belfast Health and Social Care Trust, UK

Keywords: quidditch, injury, gender, equality, concussion

https://doi.org/10.26603/001c.28225

\section{International Journal of Sports Physical Therapy}

Vol. 16, Issue 5, 2021

\section{Background}

Quidditch is a mixed-gender, full-contact sport founded in the USA in 2005, played worldwide by an estimated 25,000 players. It is one of the few mixed-gender full-contact sports, yet there remain few published studies regarding injury rates and patterns. A previous study suggested that the overall rate of injury in quidditch is in line with other contact sports, however raised concerns that female players were sustaining a higher rate of concussion when compared to male players.

\section{Purpose}

To examine injury rates and injury patterns in UK quidditch athletes over the course of a single season.

\section{Study design}

Prospective epidemiological study

\section{Methods}

Data were prospectively collected by professional first aid staff for the 2017-18 season spanning all major UK tournaments, involving 699 athletes. Anonymized player demographics were collected by an online survey. Time loss injury rates were measured per 1000 athletic exposures (AEs) and hours of play.

\section{Results}

The overall time loss injury rate was 20.5 per 1000 hours or 8.0 per 1000 AEs. The combined rate of concussion was 7.3 per 1000 hours or 2.8 per 1000 AEs. There was no statistical difference between time loss injuries in males (20.9/1000 hours and 8.1/1000 AEs) and females (13.9/1000 hours and 5.4/1000 AEs) ( $p=0.30)$ and no statistical difference between concussion rates in males $(n=7)$ and females $(n=4)(p=0.60)$.

\section{Conclusions}

Total time loss injury rates in quidditch appear to be comparable with other full-contact sports such as football. The rate of concussions for both males and females appear higher when compared to other contact sports.

\section{Level of evidence}

3

\footnotetext{
a Corresponding author: 


\section{INTRODUCTION}

Competitive quidditch is a mixed-gender, full-contact sport played worldwide. ${ }^{1}$ College students in the USA founded the real-life sport in 2005 after being inspired by the magical game of Quidditch created by JK Rowling in her Harry Potter novels. ${ }^{2}$ There are now at least 450 registered teams across the globe with 3500 registered athletes in the USA alone. ${ }^{3}$ Quidditch is played in over 40 countries by an estimated 25,000 players. ${ }^{4}$ The governing body of quidditch in the UK is QuidditchUK. 5

Rules are similar to the fictional game, involving two teams of seven players all mounted on a broomstick. Chasers aim to throw the quaffle (a deflated volleyball) through any of the three opposing hoops to score 10 points, but must negotiate their way past the keeper. ${ }^{6}$ Beaters can throw bludgers (a dodgeball) at opposing players, which temporarily suspends them from play. The snitch (a golden, winged, self-mobilizing ball in the novels) is an impartial player dressed entirely in yellow, shielding a tennis ball in a sock hanging from the back of their shorts. A seeker from each team aims to 'catch' the snitch (detach the tennis ball), which awards that team 30 points and ends the game. Players must have a broomstick between their thighs during play, but unlike in the wizarding world, are unable to levitate.

Quidditch is a fairly unique sport in that mixed-gender teams are mandatory with the rules stating that 'a team may not have more than four players who identify as the same gender in play at the same time'. 6 Quidditch therefore promotes inclusion of players of all genders, including those who do not identify with the traditional binary genders. Consequently, it is a highly progressive and inclusive team sport. However, it also poses theoretical risks of increased injury rates due to its full contact and mixed gender nature.

The QuidditchUK season consists of the Northern and Southern Regional Cups as qualifiers for the British Quidditch Cup (BQC), alongside smaller events. The Quidditch Premier League (QPL) also takes place each year and is the biggest and most successful league championship played outside the national governing body. Players who play in the QuidditchUK season also compete in the QPL.

Undertaking injury surveillance is important to ensure that risk factors can be identified, enabling preventative strategies including rule evolvement as this new sport develops. A previous retrospective study of injuries in quidditch identified several areas of interest. ${ }^{7}$ The most significant of these was that female players reported an increased incidence of concussion compared to their male teammates. This prospective injury surveillance study was designed to examine these injury trends further, and to more accurately assess the risks posed by this emerging sport. Injuries can be considered significant if they are "time loss injuries" (TL injuries) meaning the athlete is not able to continue playing that day and has a period of recovery before being able to play again. The purpose of this study is to examine injury rates and injury patterns in UK quidditch athletes over the course of a single season.

\section{METHODS}

This was a prospective observational study undertaken over the 2017 - 2018 QuidditchUK season and the QPL. Selfreported demographic data consisting of age, sex, gender, height, weight, and player position were collected using a mandatory standardised anonymous pre-competition questionnaire. This questionnaire was completed through Google forms as part of the registration process. Every competing player provided consent for the study and was therefore included.

Injury data were prospectively collected by professional first aid staff present at the events, using a standardized player-injury form at the time of injury. Information about any injury that was assessed by a first aider was recorded, regardless of severity of the injury. Details of the injury included: injury type, body location of injury, level of assessment required, treatment required and whether the player could return to competition. Player self-reported demographics were recorded again as the pre-competition data were anonymous and could not be linked to injuries. Consent was taken to store information about their demographics and injury anonymously. All data were stored using Microsoft Excel (Version 2016 MSO).

The primary injury definition used for the study was a TL injury, which was defined as any injury which resulted in an inability to return to play that day. Players who sustained injuries which were treated but did not cause a loss of playing time (e.g. minor cuts) were not included in the analysis. Concussion was defined as a "head injury with significant impact force or shows an observable sign of concussion" or "reported symptoms of concussion", as per the QuidditchUK concussion protocol (Appendix 1).

Injury rates were calculated as the incidence per 1000 hours of competitive play (/1000 hours) and per 1000 athletic exposures (/1000 AEs). The injury rates relate to match play only; no data from training sessions were included. The Pearson Chi squared test was used to compare the time loss injury rates and concussion rates between males and females using Microsoft Excel.

For the purposes of this study, sex was defined as either male or female as assigned at birth and gender was defined by the individual's self-identification. Individuals self-reported sex was used for analysis due to the wide variety of non-binary genders reported. This led to difficulties in maintaining confidentiality with small numbers of athletes in non-binary categories, and difficulty in comparing injury rates with other sports.

\section{ETHICAL APPROVAL}

The Health Research Authority online tool indicated that this study did not need National Health Service Research Ethics Committee (NHSREC) review for sites in England or Scotland. A local research ethics committee was also contacted for confirmation of this before data were collected. 
Table 1. Player demographics

\begin{tabular}{|l|l|l|l|l|}
\hline \multicolumn{9}{|c|}{ Quidditch Player Demographics } \\
\hline $\begin{array}{l}\text { Parameter } \\
\text { Mean }\end{array}$ & $\begin{array}{l}\text { Male } \\
(\mathrm{n}=378)\end{array}$ & $\begin{array}{l}\text { Female } \\
(\mathrm{n}=315)\end{array}$ & $\begin{array}{l}\text { Prefer not to say } \\
(\mathrm{n}=6)\end{array}$ & $\begin{array}{l}\text { Total } \\
(\mathrm{n}=699)\end{array}$ \\
\hline Age years & 21.7 & 21.2 & 22.8 & 21.5 \\
\hline Height $\mathrm{cm}$ & 178.7 & 171.4 & 184.7 & 175.5 \\
\hline Weight $\mathrm{kg}$ & 76.3 & 64.8 & 76.3 & 71.1 \\
\hline
\end{tabular}

Table 2. Injuries seen over the course of the season

\begin{tabular}{|c|c|c|}
\hline Type of Injury & & Frequency \\
\hline Concussion & & 11 \\
\hline Head & 11 & \\
\hline Soft tissue injury e.g. sprain/ligament injury & & 9 \\
\hline Wrist/hand & 3 & \\
\hline Shoulder & 1 & \\
\hline Knee & 2 & \\
\hline Ankle & 3 & \\
\hline Suspected fracture / joint dislocation & & 6 \\
\hline Wrist/hand & 2 & \\
\hline Shoulder & 1 & \\
\hline Ribs & 3 & \\
\hline Haematoma / Bruise & & 3 \\
\hline Wrist/hand & 1 & \\
\hline Knee & 1 & \\
\hline Ankle & 1 & \\
\hline Wound - laceration & & 2 \\
\hline Lip & 1 & \\
\hline Head & 1 & \\
\hline Total & & 31 \\
\hline
\end{tabular}

\section{RESULTS}

\section{DEMOGRAPHICS}

In the 2017 - 2018 quidditch season there were 56 teams competing in the four competitions studied, comprising 699 players. Self-reported player demographics are shown in Table 1.

The sex (as assigned to at birth) of 315 players were female and the sex of 378 players were male. Six players preferred not to disclose their sex, and thus have been excluded from the analysis. Of the 699 players, 302 identified as being a woman, 360 identified as being a man. Of these, 10 reported as being transgender (four women and six men). Twenty-nine players identified as being non-binary and eight preferred not to disclose their gender.

There were 3,868 athletic exposures equating to 1,509 hours of play. A total of 102 injuries required assessment, with 31 TL injuries identified. Of the players sustaining a TL injury, there were 9 females and 22 males. There were no significant differences in mean age, weight, height or BMI among injured athletes compared with non-injured athletes. In total, there were 11 concussions: four females and seven males. There were 12 injuries that required referral to the local accident and emergency department for assessment.

\section{INJURY TYPE}

Table 2 presents the breakdown of the injuries sustained. Head and extremity injuries were the most common injuries observed.

\section{OVERALL INJURY RATE}

The overall TL injury rate was $20.5 / 1000$ hours or $8.0 / 1000$ AEs. In males the TL injury rate was 20.9/1000 hours and 8.1/1000 AEs and in females it was 13.9/1000 hours and 5.4/ 1000 AEs. There was no statistical difference between TL injuries for males vs female $(\mathrm{p}=0.30)$, shown in Table 3 . 
Table 3. Injuries sustained by male and female subjects

\begin{tabular}{|c|c|c|c|}
\hline & Injured & Uninjured & Total \\
\hline Male & 18 & 832 & 850 \\
\hline Female & 9 & 633 & 642 \\
\hline Total & 27 & 1465 & 1492 \\
\hline \multicolumn{2}{|c|}{$\mathrm{p}=0.30$, regarding difference between time loss injuries for males vs. females } \\
\hline
\end{tabular}

Table 4. Concussions sustained by male and female subjects

\begin{tabular}{|c|c|c|c|}
\hline \multicolumn{5}{|c|}{ Concussion } \\
\hline & Concussion & No concussion & Total \\
\hline Male & 7 & 843 & 850 \\
\hline Female & 4 & 638 & 642 \\
\hline Total & 11 & 1481 & 1492 \\
\hline \multicolumn{2}{|c|}{$\mathrm{p}=0.60$, regarding difference between concussions in males and females. } \\
\hline
\end{tabular}

Table 5. Time loss injury rates in match play in other sports compared to quidditch

\begin{tabular}{|l|r|r|r|r|}
\hline & \multicolumn{7}{|c|}{ Time loss injury rates / 1000 hours } \\
\hline & Football & Lacrosse & Ice hockey & Quidditch \\
\hline Male & 18.8 & 12.6 & 16.3 & 8.1 \\
\hline Female & 16.4 & 7.2 & 12.6 & 5.4 \\
\hline
\end{tabular}

\section{CONCUSSION RATE}

The combined rate of concussion was 7.3/1000 hours or 2.8/ 1000 AEs. There was no statistical difference in concussion rate between males and females $(\mathrm{p}=0.60)$, which is shown in Table 4.

\section{MECHANISM OF CONCUSSION}

Of the 11 players that sustained a concussion injury, nine were reported as due to a tackle/body block, and two due to contact with the ground.

\section{DISCUSSION}

Quidditch is a sport that is progressive in terms of gender equality, with increasing popularity throughout the world. The nature of the sport is full-contact between mixed sexes and genders, ages and abilities. This study is the first prospective, longitudinal study looking at an entire season of competitive quidditch and will inform the governing body and participants of the sport.

It is important to note that individuals self-reported sex was used for analysis due to the wide variety of non-binary genders reported. This led to difficulties in maintaining confidentiality with small numbers of athletes in non-binary categories, and difficulty in comparing injury rates with other sports.

\section{OVERALL INJURY RATE}

Injury rates in quidditch appear to be comparable with other widely played full contact sports. For comparison, a large study undertaken by Hootman et. al. looking at the epidemiology of collegiate sporting injuries is summarized in Table 5 , showing that in other sports, TL injury rates are higher. ${ }^{8}$ Hootman focused on analyzing data for injuries that occurred during participation in an organized intercollegiate practice or contest.

\section{CONCUSSION RATE}

A previous retrospective study suggested high rates of concussion in quidditch warranting further investigation. ${ }^{7} \mathrm{~A}$ systematic review undertaken by Prien et. al. looking at the epidemiology of concussions in contact team sports has been summarized in Table 6 , to provide comparison to the rates of quidditch from this current study. ${ }^{9}$ Concussion rates in quidditch are higher than that of rugby, a sport which is often considered high risk for concussion. Reassuringly, the rates of concussion appear similar between males and females and do not suggest that allowing full contact between males and females is problematic in terms of head injuries. The previous study was based on a retrospective self-reporting questionnaire and is subject to bias in self-reporting between sexes. Broshek et. al. suggested that there were self-reporting differences in concussion between sexes which further complicates concussion reporting in mixed- 
Table 6. Concussion rates in match play compared to quidditch.

\begin{tabular}{|l|l|l|l|l|l|l|l|l|}
\hline & \multicolumn{2}{|l|}{ Football } & \multicolumn{2}{|l|}{ Rugby } & \multicolumn{2}{l|}{ Ice Hockey } & \multicolumn{3}{l|}{ Quidditch } \\
\hline & $\begin{array}{l}\mid 1000 \\
\text { hours }\end{array}$ & $\begin{array}{l}/ 1000 \\
\mathrm{AE}\end{array}$ & $\begin{array}{l}/ 1000 \\
\text { hours }\end{array}$ & $\begin{array}{l}/ 1000 \\
\mathrm{AE}\end{array}$ & $\begin{array}{l}/ 1000 \\
\text { hours }\end{array}$ & $\begin{array}{l}/ 1000 \\
\mathrm{AE}\end{array}$ & $\begin{array}{l}/ 1000 \\
\text { hours }\end{array}$ & $\begin{array}{l}/ 1000 \\
\mathrm{AE}\end{array}$ \\
\hline Male & 0.44 & 1.07 & 3.89 & 3.00 & 2.01 & 1.63 & 8.1 & 3.2 \\
\hline Female & 1.76 & 1.48 & 1.58 & 2.11 & 2.33 & 2.27 & 6.2 & 2.4 \\
\hline
\end{tabular}

sex and mixed-gender sports. ${ }^{10}$

A conference paper by Tran et. al. profiled concussion in quidditch players in Major League Quidditch, a semi-professional league based in the USA and Canada. ${ }^{11}$ In this study, twenty-five percent of players reported to have sustained a medically confirmed concussion during play. This was higher than the concussion rates found in this study. However, this study was based on an emailed retrospective self-reported survey, had a response rate of $34 \%$, and is yet to be published in a peer-reviewed journal.

Previous research led to QuidditchUK introducing a Concussion Policy, which demonstrates the sport is evolving to improve safety for participants (Appendix 1). This policy provides protocols for the diagnosis and management of suspected head injuries including the requirement of a medical opinion to authorise return to play where a diagnosis of concussion is suspected. All concussions identified in this study were TL injuries, indicating that the protocol was followed and patients with concussion were removed from play. The present study confirms relatively high rates of concussion in quidditch and the need for awareness of concussion within the quidditch community. By following the concussion policy, players are removed from play and are protected from more significant head injuries.

The finding of no significant difference in overall injury and concussion rates between males and females is reassuring. This study suggests that mixed sex contact sports can be safe for all, however further work is required to confirm this is the case in other mixed-gender full-contact sports.

\section{LIMITATIONS}

Few players sustained TL injuries, and only injuries sustained during competitive play were captured. To improve the quality of data, a larger study designed to capture data over multiple seasons and including training data would likely give the truest picture of injuries in quidditch. Players developing chronic TL injuries who did not present for assessment would not have been captured by the study methodology. We did not follow players up to determine days of sport lost due to their injury, and as such are unable to report on this outcome.

Despite these limitations, the present study offers a robust description of the incidence and nature of injuries occurring during quidditch matches in the United Kingdom.

\section{CONCLUSION}

Quidditch is an exciting, inclusive emerging sport which is rapidly growing in popularity throughout the world. This study has highlighted that although overall injury rates do not appear alarming, the rate of concussion is high compared with other sports. We have introduced a clear concussion policy to prevent further head injury in patients sustaining concussion.

It is important to identify injury patterns to raise the question of the need for rule changes to improve athlete safety. Incorporating the findings of the present study in the ongoing development of quidditch will be important to ensure its successful ongoing evolution as a safe sport.

\section{COMPETING INTERESTS}

We declare that we have no competing interests.

Submitted: November 09, 2020 CDT, Accepted: August 24, 2021 CDT 


\section{REFERENCES}

1. International Quidditch Association. What is quidditch? Accessed August 9, 2020. https://www.iqas port.com/what-is-quidditch/.

2. International Quidditch Association. History. Accessed August 9, 2020. https://www.iqasport.com/h istory/.

3. US Quidditch. About US Quidditch. Accessed August 9, 2020. https://www.usquidditch.org/about/ mission/.

4. Quidditch Premier League. What is quidditch? Accessed August 9, 2020. https://www.quidditchprem ierleague.com/quidditch-101\#: : :text=Quidditch\%20i s\%20played\%20in\%20over\%2040\%20countries\%20b y\%2025\%2C000\%20players.

5. QuidditchUK. The Quidditch UK Constitution. Accessed August 9, 2020. https://chaser.cdn.prismic.i o/chaser/5f9d200a-6134-417d-a33d-91e00c5a7b25 O UK_CONSTITUTION_DEPARTMENTEDITS_090120.pd f.

6. International Quidditch Association. The IQA Rulebook. Accessed August 9, 2020. https://www.iqas port.com/rulebook/.
7. Pennington R, Cooper A, Edmond E, Faulkner A, Reidy M, Davies P. Injuries in quidditch: a descriptive epidemiological study. Int J Sports Phys Ther. 2017;12(5):833-839.

8. Hootman J, Randall D, Agel J. Epidemiology of collegiate injuries for 15 sports: summary and recommendations for injury prevention initiatives. $J$ Athl Train. 2007;42(2):311-319.

9. Prien A, Grafe A, Rossler R, Junge A, Verhagen E. Epidemiology of head injuries focusing on concussions in team contact sports: a systematic review. Sports Med. 2018;48(4):953-969. doi:10.1007/s 40279-017-0854-4

10. Broshek DK, Kaushik T, Freeman JR, Erlanger D, Webbe F, Barth JT. Sex differences in outcome following sports-related concussion. J Neurosurg. 2005;102(5):856-863.

11. Tran V, Walser S, Wayland J, Elwood A, Posas J. Catch the snitch or the consequences? profiling incidence of concussions in quidditch players. $\mathrm{Am}$ Acad Neurol. 2018;91(23). doi:10.1212/01.wnl.000055 0653.12040.a6 


\section{SUPPLEMENTARY MATERIALS}

\section{Appendix 1. Concussion Protocol}

Download: https://ijspt.scholasticahq.com/article/28225-injuries-in-quidditch-a-prospective-study-from-a-completeuk-season/attachment/70962.docx 